\title{
COMPOSICIÓN Y TAXONOMIA DE LA FAMILIA BAETIDAE (INSECTA: EPHEMEROPTERA) PARA LA CUENCA DEL RÍO LA VIEJA, ALTO CAUCA, COLOMBIA.
}

\author{
COMPOSITION AND TAXONOMY OF THE BAETIDAE FAMILY (INSECTA: \\ EPHEMEROPTERA) FOR LA VIEJA RIVER BASIN, ALTO CAUCA, \\ COLOMBIA.
}

\author{
Luis Salinas ${ }^{1,2}$, Paola Villegas-A ${ }^{1}$ \& César Román-Valencia ${ }^{1 *}$ \\ 1Universidad del Quindío, Laboratorio de Ictiología, Grupo de Investigación en \\ "Diversidad Faunística", Armenia, Colombia. \\ 2 Laboratório de Fisiologia e Neurobiologia de Invertebrados - Departamento de \\ Entomologia, Universidade Federal de Viçosa, Brasil. \\ villegaspaolita@gmail.com, biobaetodes@gmail.com, ceroman@uniquindio.edu.co \\ *Correspondencia del Autor: ceroman@uniquindio.edu.co
}

\section{RESUMEN.}

Baetidae es una de las familias de insectos más abundantes y diversas del orden Ephemeroptera. En Colombia se registran 38 especies en 19 géneros, de las cuales cinco especies se localizan en la cuenca del Río La Vieja: Andesiops peruvianus, Camelobaetidius edmunsi, Mayobaetis ellenae, Nanomis galera y Prebaetodes sitesi. En este trabajo, se examinaron un total de 7226 ninfas de baetidos, colectadas en el Río La Vieja, pertenecientes a 23 especies en 11 géneros. Se encontró que el género más abundante y con mayor riqueza es Baetodes seguido por Camelobaetidius (10 individuos y tres especies determinadas respectivamente), las especies más abundantes fueron Baetodes awa y Camelobaetidius edmunsi (con 3563 y 1282 individuos respectivamente). En el presente trabajo se registra por primera vez, para el Río La Vieja, dos géneros (Cloeodes y Varipes) y ocho especies (Baetodes awa, Baetodes diasae, Baetodes pseudospinae, Camelobaetidius mathuriae, Camelobaetidius patricki, Nanomis rasmusseni, Prebaetodes meridinensis y Zelusia principales). Lo anterior establece que el rio La Vieja registra alrededor del $61 \%$ de las especies de baetidos reconocidas para Colombia (23 de las 38 especies).

Palabras claves: Artrópoda, Taxonomía, Comunidades, Andes, Sur América.

Cómo citar:

Salinas, L., Villegas-A, P \& Román-Valencia, C.,(2019) COMPOSICIÓN Y TAXONOMIA DE LA FAMILIA BAETIDAE (INSECTA: EPHEMEROPTERA) PARA LA CUENCA DEL RÍO LA VIEJA, ALTO CAUCA, COLOMBIA. Revista de Investigaciones Universidad del Quindío. Vol 31, pp.15-25. 


\begin{abstract}
.
Baetidae is one of the most abundant and diverse families in the insect order Ephemeroptera. Among the baetid species distributed in Colombia (38 species and 9 genera), five are known to occur in the basin of La Vieja river, Alto Cauca (Andesiops peruvianus, Camelobaetidius edmunsi, Mayobaetis ellenae, Nanomis gallera, and Prebaetodes sitesi). Here, we estimate the species richness of Baetidae in La Vieja river based on specimens deposited in local museums. We examined 7226 baetid nymphs from 23 species in 11 genera collected in the La Vieja river. We found Baetodes and Camelobaetidius to be the richest genera (10 and three species, respectively), with Baetodes awa and Camelobaetidius edmunsi being the most abundant species in the river (3 563 and 1 282 individuals respectively). Here we present new registries for two genera (Cloeodes and Varipes) and eight species (Baetodes awa, Baetodes diasae, Baetodes pseudospinae, Camelobaetidius mathuriae, Camelobaetidius patricki, Nanomis rasmusseni, Prebaetodes meridinensis, and Zelusia principalis) in La Vieja river. Our richness estimates indicates that La Vieja river harbors around $61 \%$ of the baetid species described in Colombia (23 of the 38 species).
\end{abstract}

Key words: Arthropoda, Community, Taxonomy, Andean, South America.

\section{INTRODUCCIÓN.}

La Familia Baetidae fue establecida en 1815 por W.E. Leach (Domínguez et al., 2006) y comprende uno de los mayores grupos de Ephemeroptera (Salles et al., 2004). Los baetidos están representados por 140 géneros y 900 especies aproximadamente, presentan una distribución cosmopolita con excepción en la Antártica y Nueva Zelandia (Lugo-Ortiz \& McCafferty, 1999; Gattolliat \& Nieto, 2009). En Sur América, esta familia representa la mayor riqueza con 175 géneros y 34 especies registradas (Domínguez \& Dos Santos, 2014). En Colombia se encuentran registrados aproximadamente 19 géneros y 38 especies (Días et al., 2009; Gutiérrez \& Reinoso, 2010; Salinas et al., 2011; 2012; 2017; 2018; Motta-Díaz et al., 2012; Rozo \& Salinas, 2016; Vinasco-Mondragón \& Zúñiga, 2016), de las cuales nueve géneros (Americabaetis, Andesiops, Baetodes, Callibaetis, Camelobaetidius, Mayobaetis, Nanomis, Prebaetodes, Zelusia) y cinco especies (Andesiops peruvianus, Camelobaetidius edmunsi, Mayobaetis ellenae, Nanomis galera y Prebaetodes sitesi) han sido reportadas para la cuenca del río La Vieja, Alto Cauca (Días et al., 2009; Zúñiga et al., 2013).

La cuenca del río La Vieja ha sufrido transformaciones en sus ecosistemas naturales debido al consecuente aumento de actividades tales como la agricultura intensiva, la minería, extracción de material de río y la ganadería (Zúñiga et al., 2013; Román-P \& Román-Valencia, 2017; García-Alzate et al., 2017; Ruiz-C. et al., 2018). Lo que provocan impactos negativos sobre las características de los ambientes acuáticos, produciendo efectos sobre la diversidad de la fauna béntica (Chará et al., 2009). Por lo cual es útil y necesario realizar estudios enfocados en la sistemática y ecología de aquellos grupos para los cuales existe un gran vacío en su reconocimiento (Zúñiga et al., 2013), y permiten obtener información básica como línea que sustente estrategias de prevención, conservación y de restauración (Ruiz-C. et al., 2018). En este sentido, el objetivo de éste trabajo fue realizar un reconocimiento taxonómico de la familia Baetidae para la cuenca del río la Vieja en Alto Cauca, Colombia.

\section{MATERIAL Y MÉTODOS.}

Área de estudio: El río la Vieja es el resultado de la confluencia de los ríos Barragán y Quindío. Es uno de los principales tributarios del Alto río Cauca y su cuenca hidrográfica está ubicada en el centro-occidente de Colombia en la jurisdicción de los departamentos del Quindío, Risaralda y Valle del Cauca. Se enmarca en las coordenadas: $4^{\circ} 04^{\prime}$ y $4^{\circ} 49^{\prime}$ de latitud norte y $75^{\circ} 24^{\prime}$ y $75^{\circ} 57^{\prime}$ de longitud oeste. Su extensión es de $2880 \mathrm{Km}^{2}$ correspondiéndole el $68 \%$ al departamento del Quindío (1 $\left.961 \mathrm{Km}^{2}\right)$, el 10\% al departamento de Risaralda $\left(298 \mathrm{Km}^{2}\right)$ y el $22 \%$ al departamento del Valle del Cauca $\left(619 \mathrm{Km}^{2}\right)$ (Román-P \& Román-Valencia, 2017; García-Alzate et al., 2017). 
Fase de laboratorio: Se examinaron 7226 ninfas de la familia Baetidae preservados en alcohol al 96\% y depositadas en las colecciones de macroinvertebrados del laboratorio de Ictiología (MIUQ), de Insectos (CIUQ) y del Museo de Artrópodos (MAUQ) en la Universidad del Quindío, Armenia, Colombia. El material revisado fue colectado en 44 puntos de muestreo (P1 a P44) en 23 corrientes hídricas (quebradas), las cuales cubrieron un rango altitudinal entre 984 hasta 3076 metros sobre el nivel del mar (msnm), pertenecientes a la cuenca del río La Vieja, Alto Cauca (Tabla 1).
Para la determinación de las muestras examinadas fueron utilizadas las claves taxonómicas de Domínguez et al. (2006) y Domínguez et al. (2009). Se realizaron montajes permanentes de las estructuras de importancia taxonómica como patas, piezas bucales y paraproctos, así se utilizó la metodología de Waltz \& McCafferty (1987), se utilizaron cubreobjetos, portaobjetos y Euparal. Las observaciones se efectuaron en un microscopio Olympus Modelo CX21 y un estereoscopio Zeiss Stemi DV4. La toma de fotografías, para registros, se realizó con estereoscopio Zeiss Stemi 2000c con cámara Axio Cam ERc 5s realizado con el programa Zen 2 lite.

Tabla 1. Sitios de colecta (P1 a P44), altura (msnm), coordenadas geográficas, nombre del drenaje (Quebrada, Río) y Acronimia de colección (MIUQ: Macroinvertebrados del Ictiología Universidad del Quindío, MAUQ: Museo de Artrópodos de la Universidad del Quindío; CIUQ: Colección de Insectos de la Universidad del Quindío). S. D.: Santo Domingo; Q1 a Q4: Quebrada 1 a la 4; RN: Reserva Natural.

\begin{tabular}{|c|c|c|c|c|}
\hline $\begin{array}{l}\text { Sitio de } \\
\text { Colecta }\end{array}$ & Altura & Coordenadas & $\begin{array}{l}\text { Drenaje (Río o } \\
\text { Quebrada) }\end{array}$ & Colección \\
\hline $\mathbf{P 1}$ & 1892 & $4^{\circ} 38^{\prime} 32.46^{\prime \prime} \mathrm{N}, 75^{\circ} 33^{\prime} 28.29^{\prime \prime} \mathrm{O}$ & Río Quindío & MIUQ \\
\hline P2 & 1773 & $4^{\circ} 37^{\prime} 29.16^{\prime \prime} \mathrm{N}, 75^{\circ} 34^{\prime} 35.98^{\prime \prime} \mathrm{O}$ & Río Navarco & MIUQ \\
\hline P3 & 1764 & $4^{\circ} 38^{\prime} 34.39^{\prime \prime} \mathrm{N}, 75^{\circ} 35^{\prime} 1.13^{\prime \prime} \mathrm{O}$ & Río Quindío & MIUQ \\
\hline P4 & 1868 & $4^{\circ} 38^{\prime} 9.16^{\prime \prime} \mathrm{N}, 75^{\circ} 36^{\prime} 56.71^{\prime \prime} \mathrm{O}$ & Quebrada El Bosque & MIUQ \\
\hline P5 & 1646 & $4^{\circ} 37^{\prime} 28.75^{\prime \prime} \mathrm{N}, 75^{\circ} 38^{\prime} 44.82^{\prime \prime} \mathrm{O}$ & Río Roble & MAUQ \\
\hline P6 & 1484 & $4^{\circ} 37^{\prime} 34.92^{\prime \prime} \mathrm{N}, 75^{\circ} 40^{\prime} 33.94^{\prime \prime} \mathrm{O}$ & Río Roble & MIUQ \\
\hline P7 & 1400 & $04^{\circ} 35^{\prime} 43.7^{\prime \prime} \mathrm{N}, 75^{\circ} 42^{\prime} 44.62^{\prime \prime} \mathrm{O}$ & Río Roble & MAUQ \\
\hline P8 & 1160 & $04^{\circ} 34^{\prime} 20.43^{\prime \prime} \mathrm{N}, 75^{\circ} 48^{\prime} 0.86^{\prime \prime} \mathrm{O}$ & Río Roble & MAUQ \\
\hline P9 & 986 & $04^{\circ} 34^{\prime} 43.91^{\prime \prime} \mathrm{N}, 75^{\circ} 51^{\prime} 21.86^{\prime \prime} \mathrm{O}$ & Río Roble & CIUQ \\
\hline P10 & 984 & $04^{\circ} 34^{\prime} 46.06^{\prime \prime} \mathrm{N}, 75^{\circ} 51^{\prime} 22.74^{\prime \prime} \mathrm{O}$ & Río Roble & MIUQ \\
\hline P11 & 1265 & $04^{\circ} 33^{\prime} 25.1^{\prime \prime} \mathrm{N}, 75^{\circ} 42^{\prime} 44.62^{\prime \prime} \mathrm{O}$ & $\begin{array}{l}\text { Quebrada afluente Rio } \\
\text { Roble }\end{array}$ & MAUQ \\
\hline P12 & 1244 & $04^{\circ} 29^{\prime} 53.33^{\prime \prime} \mathrm{N}, 75^{\circ} 48^{\prime} 0.16^{\prime \prime} \mathrm{O}$ & Río Espejo & MAUQ \\
\hline P13 & 1054 & $04^{\circ} 23^{\prime} 53.75^{\prime \prime} \mathrm{N}, 75^{\circ} 48^{\prime} 12.26^{\prime \prime} \mathrm{O}$ & Río La Vieja & CIUQ \\
\hline P14 & 1054 & $04^{\circ} 24^{\prime} 27.27^{\prime \prime} \mathrm{N}, 75^{\circ} 47^{\prime} 45.85^{\prime \prime} \mathrm{O}$ & Quebrada Bosque & CIUQ \\
\hline P15 & 1480 & $04^{\circ} 34^{\prime} 27^{\prime \prime} \mathrm{N}, 75^{\circ} 40^{\prime} 11^{\prime \prime} \mathrm{O}$ & Quebrada Hojas Anchas & MIUQ \\
\hline P16 & 1465 & $04^{\circ} 33^{\prime} 10^{\prime \prime} \mathrm{N}, 75^{\circ} 40^{\prime} 22^{\prime \prime} \mathrm{O}$ & Quebrada Hojas Anchas & MIUQ \\
\hline P17 & 1243 & $04^{\circ} 32^{\prime} 23^{\prime \prime} \mathrm{N}, 75^{\circ} 44^{\prime} 11^{\prime \prime} \mathrm{O}$ & Quebrada Hojas Anchas & MIUQ \\
\hline P18 & 1240 & $04^{\circ} 32^{\prime} 21^{\prime \prime} \mathrm{N}, 75^{\circ} 44^{\prime} 27^{\prime \prime} \mathrm{O}$ & Quebrada Hojas Anchas & MIUQ \\
\hline P19 & 1750 & $04^{\circ} 30^{\prime} 36.5^{\prime \prime} \mathrm{N}, 75^{\circ} 36^{\prime} 48.2^{\prime \prime} \mathrm{O}$ & Río Santo Domingo & MIUQ \\
\hline $\mathbf{P 2 0}$ & 1684 & $04^{\circ} 30^{\prime} 34.39^{\prime \prime} \mathrm{N}, 75^{\circ} 36^{\prime} 49.41^{\prime \prime} \mathrm{O}$ & Río Santo Domingo & MIUQ \\
\hline $\mathbf{P 2 1}$ & 1626 & $04^{\circ} 30^{\prime} 45.2^{\prime \prime} \mathrm{N}, 75^{\circ} 36^{\prime} 47.1^{\prime \prime} \mathrm{O}$ & Río Santo Domingo & MIUQ \\
\hline $\mathbf{P 2 2}$ & 1636 & $04^{\circ} 31^{\prime} 07.9^{\prime \prime} \mathrm{N}, 75^{\circ} 36^{\prime} 53.1^{\prime \prime} \mathrm{O}$ & Río Santo Domingo & MIUQ \\
\hline
\end{tabular}




\begin{tabular}{|c|c|c|c|c|}
\hline $\mathbf{P 2 3}$ & 1560 & $04^{\circ} 31^{\prime} 16.5^{\prime \prime} \mathrm{N}, 7^{\circ} 37^{\prime} 05.2^{\prime \prime} \mathrm{O}$ & Río Santo Domingo & MIUQ \\
\hline P24 & 1269 & $04^{\circ} 27^{\prime} 02.7^{\prime \prime} \mathrm{N}, 7^{\circ} 41^{\prime} 22.7^{\prime \prime} \mathrm{O}$ & Río Santo Domingo & MIUQ \\
\hline $\mathbf{P 2 5}$ & 1283 & $04^{\circ} 27^{\prime} 03.5^{\prime \prime} \mathrm{N}, 75^{\circ} 41^{\prime} 29.6^{\prime \prime} \mathrm{O}$ & Río Santo Domingo & MIUQ \\
\hline $\mathbf{P 2 6}$ & 1243 & $04^{\circ} 26^{\prime} 50.5^{\prime \prime} \mathrm{N}, 75^{\circ} 41^{\prime} 28.1^{\prime \prime} \mathrm{O}$ & Río Santo Domingo & MIUQ \\
\hline $\mathbf{P 2 7}$ & 1265 & $04^{\circ} 26^{\prime} 45.2^{\prime \prime} \mathrm{N}, 75^{\circ} 41^{\prime} 33.5^{\prime \prime} \mathrm{O}$ & Río Santo Domingo & MIUQ \\
\hline $\mathbf{P 2 8}$ & 1166 & $04^{\circ} 24^{\prime} 38.2^{\prime \prime} \mathrm{N}, 75^{\circ} 43^{\prime} 14.2^{\prime \prime} \mathrm{O}$ & Río Santo Domingo & MIUQ \\
\hline P29 & 1145 & $04^{\circ} 24^{\prime} 31.4^{\prime \prime} \mathrm{N}, 75^{\circ} 43^{\prime} 19.3^{\prime \prime} \mathrm{O}$ & Río Santo Domingo & MIUQ \\
\hline P30 & 1143 & $04^{\circ} 24^{\prime} 31.5^{\prime \prime} \mathrm{N}, 75^{\circ} 43^{\prime} 29.9^{\prime \prime} \mathrm{O}$ & Río Santo Domingo & MIUQ \\
\hline P31 & 1140 & $04^{\circ} 24^{\prime} 26.2^{\prime \prime} \mathrm{N}, 75^{\circ} 43^{\prime} 28.5^{\prime \prime} \mathrm{O}$ & Río Santo Domingo & MIUQ \\
\hline P32 & 2850 & $04^{\circ} 25^{\prime} 23.04^{\prime \prime} \mathrm{N}, 75^{\circ} 36^{\prime} 43.76^{\prime \prime} \mathrm{O}$ & $\begin{array}{l}\text { Quebrada 1, Río Santo } \\
\text { Domingo. }\end{array}$ & MAUQ \\
\hline P33 & 2763 & $04^{\circ} 26^{\prime} 39^{\prime \prime} \mathrm{N}, 75^{\circ} 37^{\prime} 24^{\prime \prime} \mathrm{O}$ & $\begin{array}{l}\text { Quebrada 2, Río Santo } \\
\text { Domingo. }\end{array}$ & MAUQ \\
\hline P34 & 2065 & $04^{\circ} 32^{\prime} 40.06^{\prime \prime} \mathrm{N}, 75^{\circ} 35^{\prime} 40.38^{\prime \prime} \mathrm{O}$ & $\begin{array}{l}\text { Quebrada 3, Río Santo } \\
\text { Domingo. }\end{array}$ & MAUQ \\
\hline P35 & 1300 & $04^{\circ} 30^{\prime} 7.45^{\prime \prime} \mathrm{N}, 75^{\circ} 41^{\prime} 15.06 " \mathrm{O}$ & $\begin{array}{l}\text { Quebrada 4, Río Santo } \\
\text { Domingo. }\end{array}$ & MAUQ \\
\hline P36 & 3076 & $04^{\circ} 25^{\prime} 56.4^{\prime \prime} \mathrm{N}, 75^{\circ} 37^{\prime} 29.4 ” \mathrm{O}$ & Quebrada 1 en Sonadora & CIUQ \\
\hline P37 & 3000 & $04^{\circ} 26^{\prime} 37.2^{\prime \prime} \mathrm{N}, 7^{\circ} 37^{\prime} 46.5^{\prime \prime} \mathrm{O}$ & Quebrada 2 en Sonadora & CIUQ \\
\hline P38 & 2915 & $04^{\circ} 25^{\prime} 27.6^{\prime \prime} \mathrm{N}, 75^{\circ} 36^{\prime} 36.1^{\prime \prime} \mathrm{O}$ & Quebrada El Cedral & CIUQ \\
\hline P39 & 2900 & $04^{\circ} 25^{\prime} 37.3^{\prime \prime} \mathrm{N}, 75^{\circ} 36^{\prime} 40.3^{\prime \prime} \mathrm{O}$ & Quebrada 3 en Sonadora & CIUQ \\
\hline $\mathbf{P 4 0}$ & 2800 & $04^{\circ} 26^{\prime} 37.2^{\prime \prime} \mathrm{N}, 7^{\circ} 37^{\prime} 46.2^{\prime \prime} \mathrm{O}$ & Quebrada La Sonadora & CIUQ \\
\hline P41 & 1489 & $4^{\circ} 29^{\prime} 47^{\prime \prime} \mathrm{N}, 75^{\circ} 38^{\prime} 39^{\prime \prime} \mathrm{O}$ & Quebrada El Salado & CIUQ \\
\hline P42 & 1433 & 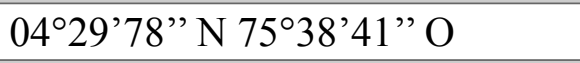 & Quebrada La Honda & CIUQ \\
\hline $\mathbf{P 4 3}$ & 1399 & $04^{\circ} 21^{\prime} 42.77^{\prime \prime} \mathrm{N} 75^{\circ} 44^{\prime} 18.18^{\prime \prime} \mathrm{O}$ & Quebrada La Cabaña & CIUQ \\
\hline P44 & 1249 & $4^{\circ} 23^{\prime} 00^{\prime \prime} \mathrm{N} 75^{\circ} 45^{\prime} 00^{\prime \prime} \mathrm{O}$ & Quebrada Sardineros & MIUQ \\
\hline
\end{tabular}

\section{RESULTADOS Y DISCUSIÓN}

Se encontraron 11 géneros y 23 especies de la familia Baetidae para la cuenca del río La Vieja (Tablas 1 y 2). En cuanto la riqueza y abundancia, el género más abundante y con mayor riqueza fue Baetodes representado por 5269 individuos y 10 especies, seguido por Camelobaetidius con 1289 individuos y tres especies. Las especies más abundantes fueron Baetodes awa y Camelobaetidius edmunsi con 3563 y 1282 individuos respectivamente (Tabla 2).

Los géneros Cloeodes y Varipes y las especies Baetodes awa, Baetodes diasae, Baetodes pseudospinae, Camelobaetidius mathuriae, Camelobaetidius patricki, Nanomis rasmusseni, Prebaetodes meridinensis y Zelusia principalis se registran por primera vez para la región, se amplía así su distribución geográfica en Colombia. 
Tabla 2. Riqueza y abundancia de especies de la Familia Baetidae de la cuenca alta del río La Vieja, Alto Cauca. Los números corresponden a la abundancia de cada taxón.

\begin{tabular}{|c|c|c|c|c|c|}
\hline \multirow[b]{2}{*}{ Género } & \multirow[b]{2}{*}{ Especies } & \multicolumn{3}{|c|}{ Abundancia por Colecciones } & \multirow{2}{*}{$\begin{array}{l}\text { Abundancia } \\
\text { Total }\end{array}$} \\
\hline & & MIUQ & MAUQ & CIUQ & \\
\hline Americabaetis & Americabaetis sp. & 19 & 19 & 3 & 41 \\
\hline Andesiops & A. peruvianus & 16 & 0 & 358 & 374 \\
\hline \multirow[t]{10}{*}{ Baetodes } & B. awa & 3563 & 0 & 0 & 3563 \\
\hline & B. diasae & 4 & 4 & 265 & 273 \\
\hline & B.pseudospinae & 45 & 8 & 5 & 58 \\
\hline & Baetodes sp. 1 & 1092 & 2 & 0 & 1094 \\
\hline & Baetodes sp. 2 & 0 & 1 & 0 & 1 \\
\hline & Baetodes sp. 3 & 77 & 0 & 0 & 77 \\
\hline & Baetodes sp. 4 & 2 & 0 & 0 & 2 \\
\hline & Baetodes sp. 5 & 4 & 0 & 0 & 4 \\
\hline & Baetodes sp. 6 & 196 & 0 & 0 & 196 \\
\hline & Baetodes sp. 7 & 0 & 0 & 1 & 1 \\
\hline Callibaetis & Callibaetis sp. & 0 & 0 & 1 & 1 \\
\hline \multirow{3}{*}{$\begin{array}{l}\text { Camelobaeti- } \\
\text { dius }\end{array}$} & C. edmunsi & 1279 & 2 & 1 & 1282 \\
\hline & C. mathuriae & 1 & 0 & 0 & 1 \\
\hline & C. patricki & 1 & 0 & 2 & 3 \\
\hline Cloeodes & Cloeodes sp. & 6 & 0 & 1 & 7 \\
\hline Mayobaetis & M. ellenae & 17 & 5 & 41 & 63 \\
\hline \multirow{2}{*}{ Nanomis } & & 4 & 0 & 0 & 4 \\
\hline & N. rasmusseni & 116 & 1 & 3 & 120 \\
\hline Prebaetodes & P. meridinensis & 0 & 1 & 49 & 50 \\
\hline Varipes & Varipes sp. & 9 & 0 & 0 & 9 \\
\hline \multirow[t]{2}{*}{ Zelusia } & Z. principales & 2 & 0 & 0 & 2 \\
\hline & Abundancia Total & 6453 & 43 & 730 & 7226 \\
\hline
\end{tabular}

Diagnosis de los taxones determinados:

\section{Americabaetis Kluge 1992}

Diagnosis: 1) Cabeza más larga que ancha; 2) Quilla frontal ausente o presente; 3) Labro dorsalmente con un par de setas subapicales mediales, margen apical con dos tipos de setas: bífidas cerca de la línea media y ramificadas cerca del margen lateral; 4) Mandíbulas con setas entre la prosteca y la mola, incisivos fusionados apicalmente, prosteca robusta con dentículos apicales; 5) Hipofaringe con lingua subigual a la superlingua;
6) Maxila con palpos bi-segmentados; 7) Labio: glosa $0,6-0,8$ veces la longitud y mitad del ancho de la paraglosa, glosa con una hilera de largas setas espiniformes, paraglosa rectangular con tres hileras de largas setas espiniformes, segmento II del palpo con una proyección distomedial; 8) $\mathrm{Pa}$ tas robustas, superficie dorsal del fémur con una hilera de espinas, tibia 1,41,5 veces el largo del tarso; 9) Uñas tarsales con una hilera simple de dentículos; 10) Pterotecas posteriores ausentes; 11) Branquias abdominales presentes y alargadas en los segmentos IIVII; 12) Margen posterior del paraprocto con espinas; y 13) Filamento terminal 
0,8-1,0 veces la longitud del cerco (Domínguez et al., 2006).

Material Examinado: MIUQ 430, 780 P28; MIUQ 716, P24; MIUQ 818, P31; MIUQ 928, P17.

Comentarios. Americabaetis posee 12 especies descritas para América del Sur (Domínguez y Dos Santos, 2014). En Colombia se encuentra reportada A. alphus (Gutiérrez y Llano, 2015). La identidad hasta la categoria especie no se pudo realizar debido al deterioro de los individuos examinados.

\section{Andesiops peruvianus Ulmer 1920 (Figura 1b.)}

Diagnosis: 1) Cabeza más larga que ancha; 2) Antenas largas, tres veces el tamaño de la cabeza; 3) Mandíbula izquierda: dentículos molares sin constricciones; 4) Lingua con una proyección apical aguda; 5) Labio no compacto; 6) Uñas tarsales con una hilera doble de dentículos, la segunda hilera con casi el mismo número de dentículos que la primera, aunque reducidas en tamaño; 7) Filamento terminal 0.65 a 0.70 veces la longitud de los cercos; 8) Margen posterior de los tergos abdominales con pequeñas espinas; 9) Branquias con poca nervadura (Domínguez et al., 2006).

\section{Material Examinado: CIUQ 8417, P38; CIUQ 8406, P40.}

Comentarios. Esta especie se conoce con base en ninfas y adultos, se registra para Argentina, Perú y Colombia (Domínguez et al., 2006). En Colombia se encuentra ampliamente en la región andina (Días et al., 2009).

\section{Baetodes awa Salinas 2011}

Diagnosis: 1) Labro con una hilera subapical de 6 cerdas continuas y largas de tamaños subiguales; 2) Dos cerdas pectinadas de diferente longitud en la parte apical de la glosa; 3) Cada coxa con dos branquias digitiformes; 4) Margen dorsal de los fémures con una hilera de cerdas largas y finas alternando con 4-6 cerdas robustas clavadas y largas, longitud mayor de la mitad de las cerdas finas; 5) Uñas tarsales con una hilera de 6-8 dentículos, los basales más pequeños que los distales, aumentando de tamaño en dirección al ápice; 6) Tubérculos abdominales presentes pero muy reducidos (Salinas et al., 2011).

Material Examinado: MIUQ 1023, P28; MIUQ 408, 572, P11; MIUQ 408, P27; MIUQ 097,120 \& 094, P26; MIUQ 107,144, 162, 195, 220,245, P15; MIUQ 155, P26; MIUQ 170,227, P16; MIUQ 174,187, 207, 216, 233,390, P17; MIUQ 201,202, P16; MIUQ 298, P44; MIUQ 298, P 44; MIUQ 362,1145, P24; MIUQ 353,496,P22; MIUQ 374, P25; MIUQ446,P30; MIUQ 460,P31; MIUQ 469, P19; MIUQ 485,1080, P21; MIUQ511,P23;618,715, P29; MIUQ 12, P31; MIUQ 739, 918, P17;MIUQ 265, P19.

Comentarios. Esta especie fue descrita a partir de ninfas. Actualmente, se encuentra registrada para Colombia (Salinas et al., 2011). B. yuracare, también posee pequeños tubérculos abdominales y dos branquias coxales, así como $B$. awa, sin embargo, ésta especie se puede distinguir de B. yuracare por el número de setas en la parte dorsal del labro (cinco en B. yuracare y seis en B. awa), la forma del ápice de la lingua (redondeado en $B$. yuracare y agudo en $B$. awa), el número y la longitud de las setas clavadas en el borde dorsal de los fémures (6-7 en B. yuracare y 4-6 en $B$. $a w a)$. Su presencia en el Rio La Vieja amplia el rango de distribución en la región occidental de Colombia.

\section{Baetodes diasae Salinas 2011}

Diagnosis: 1) Labro con una hilera subapical de cerdas compuesta con $3+13$ cerdas largas de tamaños subiguales; 2) Glosa con dos setas pectinadas; 3) Branquias coxales simples con una proyección; 4) Margen dorsal de los fémures con una hilera de cerdas cortas, finas y simples alternan con 7-8 cerdas robustas clavadas y cortas; 5) Uña tarsal con 6-7 dentículos, los basales más peque- 
ños que los distales, aumentando de tamaño en dirección al ápice; 6) Tubérculos abdominales punteados y dirigidos posteriormente (Salinas et al., 2011).

Material Examinado: MIUQ 1288, P2; MIUQ 8412, P38; MIUQ 45C, P35; MIUQ 80A, P32; MIUQ298B, P44.

Comentarios. Esta especie fue descrita a partir de ninfas. Actualmente, se encuentra registrada para Colombia (Salinas et al., 2011). Otras seis especies de Baetodes poseen branquias coxales simples (B. peniculus; B. uruguay; B. santatere$z a$; B. liviae; $B$. edmundsi y $B$. noventus). B. diasae difiere de estas especies porque es la única especie que tiene branquias coxales con una proyección basal. Su presencia en el Río La Vieja, amplia el rango de distribución en la región occidental de Baetodes pseudospinae Salinas 2011

Diagnosis: 1) Labro con una hilera subapical de cerdas compuesta con $2+4$ cerdas largas de tamaños subiguales, y presenta algunas setas mayores; 2) Glosa con una seta pectinada; 3) Cuatro tubérculos en el pronoto y dos tubérculos en el mesonoto; 4) Tubérculo prominente en el metanoto, más grande que el tubérculo del primer segmento abdominal; 5) Branquias coxales dobles; 6) Margen dorsal de los fémures con una hilera de cerdas largas y finas alternando con 9-10 cerdas robustas clavadas y largas, de longitud mayor a la mitad de las cerdas finas; 7) Uña tarsal con 6-7 dentículos, los basales más pequeños que los distales, aumentan de tamaño en dirección al ápice; 8) Tubérculos abdominales presentes y prominentes (Salinas et al., 2011).

Material Examinado: MIUQ 107C, P15; MIUQ 195C, MIUQ 239C, P15; MAUQ 267C, P5; MIUQ 353C, MIUQ 496B, P22; MIUQ 408C, P27; MIUQ 1288, P2; MIUQ1023B, P28; MIUQ 1029B, P23; MIUQ 1038C, P19.

Comentarios. Esta especie fue descrita a partir de ninfas. Actualmente, se encuentra registrada en Colombia (Salinas et al., 2011). B. pseudospi- nae evidencia algunas afinidades con $B$. spinae. Estas especies poseen dos tubérculos en pronoto y el mesonoto, además de dos branquias en cada coxa y tubérculos prominentes en el abdomen. Sin embargo $B$. pseudospinae se puede distinguir de $B$. spinae por la presencia de un tubérculo prominente en el metanoto y de una seta pectinada en el ápice de la glosa (B. spinae posee un tubérculo reducido en el metanoto y dos setas pectinadas en el ápice de la glosa). Su presencia en el Rio La Vieja, amplia el rango de distribución en la región occidental de Colombia.

\section{Callibaetis Eaton 1881}

Diagnosis: 1) Cabeza más larga que ancha; 2) Antenas largas, 5-6 veces la longitud de la cabeza; 3) Labro más ancho que largo, con una hilera de setas como espinas aplanadas a lo largo del margen anterior; 4) las mandíbulas presentan incisivos hendidos profundamente, pueden presentar o no un mechón de setas entre la prosteca y la mola; 5) Mandíbula izquierda: dos dentículos apicales en la prosteca; 6) Mandíbula derecha: con la prosteca reducida a una seta simple; 7) Hipofaringe: con lingua más larga que la superlingua y con una proyección apical redondeada, superlingua redondeada apicalmente; 8) Maxilas con largos caninos y una fila de setas largas en el ápice de la galea-lacinia, el palpo maxilar bisegmentado y entre 1 y 1,5 veces más largo que la galea-lacinia, con setas como espinas combinadas con setas simples; 9) Labio con la glosa igual o más corta pero, siempre más ancha que la paraglosa, glosa y paraglosa con una fila de setas como espinas, el segmento II del palpo labial sin proyección y con una fila de setas como espinas, el segmento III es alargado y presenta una concavidad media; 10) Patas largas y estrechas, borde dorsal de los fémures paralelo al borde ventral, borde dorsal con una fila de cortas espinas, la tibia es alargada subigual al tarso y con espinas bipectinadas; 11) margen ventral del fémur I con una fila de 2 a 3 espinas puntiagudas; 12) Uña tarsal con dos filas de dentículos cilíndricos muy largos; 13) Con pterotecas posteriores presentes; 14) Margen posterior de los tergos abdominales 
con espinas; 15) Branquias presentes en los segmentos I-VII alargadas, con la tráquea pigmentada y las dos lamelas en estrecho contacto; el margen posterior de los paraproctos presenta espinas y el filamento terminal es de similar longitud a los cercos (Domínguez et al., 2006).

Material Examinado: CIUQ 8380, CIUQ 8352, P9.

Comentarios. Callibaetis posee 26 especies descritas para América del Sur (Domínguez y Dos Santos, 2014; Vilela Cruz et al, 2014; Vilela Cruz et al, 2017). En Colombia se encuentran reportadas las especies $C$. radiatus y $C$. viviparus (Vinasco-Mondragon \& Zúñiga, 2016). La identificación a nivel especifico no se pudo realizar debido que únicamente se analizaron ninfas y la asociación con adultos es indispensable para la determinación taxonómica a esta categoría.

\section{Camelobaetidius edmundsi Dominique, Tho- mas \& Mathuriau 2006}

Diagnosis: 1) Labro dorsalmente con un par de setas cerca de la línea media subapical y 2-3 setas cerca del margen lateral; 2) Segmento del II palpo labial con una fuerte proyección distomedial redondeada; 3) Uñas tarsales con 34 a 39 dentículos; 4) Branquias coxales ausentes; 5) Filamento caudal de igual tamaño que los cercos (Domínguez et al., 2006).

Material Examinado: MIUQ 1062A, P22; MIUQ 046, P10; MIUQ 163, P15; MIUQ 202A, P16; MIUQ 669, P21.

Comentarios. Esta especie es conocida a partir de ninfas. Actualmente se encuentra registrada para Colombia y Venezuela (Domínguez et al., 2006; Días et al, 2009; Salinas et al, 2012; Vilela Cruz et al, 2012; Salinas et al, 2017).

\section{Camelobaetidius mathuriae Dominique \& Tho- mas 2006}

Diagnosis: 1) Labro dorsalmente con un par de setas subapicales cerca de la línea media y con 3-4 setas cerca al margen lateral; 2) Segmento II del palpo labial con una proyección distomedial redondeada; 3) Branquias coxales cortas; 4) Uñas tarsales con 25 a 28 dentículos; 5) Branquias abdominales con ramificación levemente oscurecido; 6) Filamento terminal largo, subigual a la longitud de los cercos (Domínguez et al., 2006).

Material Examinado: MIUQ 1062A, P22.

Comentarios. Esta especie es conocida a partir de ninfas. Actualmente se encuentra registrada para Venezuela y Colombia (Domínguez et al., 2006). C. mathuriae está estrechamente relacionado con $C$. edmundsi. Sin embargo, las ninfas de la primera especie pueden distinguirse por la presencia de branquias torácicas en la base de la coxa anterior, mientras que en $C$. edmundsi, están ausentes. Camelobaetidius patricki Dominique \& Thomas, 2006.

Diagnosis: 1) Labro dorsalmente con un par de setas subapicales cerca de la línea media y 3-4 setas lateralmente; 2) Segmento II del palpo labial con una proyección distomedial redondeada; 3) Uñas tarsales con 9 dentículos; 4) Coxas branquiales presentes y cortas, casi igual a la longitud de la coxa; 5) Branquias abdominales con tráquea principal y ramas laterales oscurecidas; 6) Filamento caudal casi igual a longitud de los cercos (Domínguez et al., 2006).

Material Examinado: CIUQ 8381, P 43.

Comentarios. Esta especie es conocida a partir de ninfas. Actualmente se encuentra registrada para Colombia (Domínguez et al., 2006).

\section{Cloeodes Traver, 1938}

Diagnosis: 1) Antena más larga que la cabeza; 2) maxila con palpo más corto o casi igual a la longitud de la galea-lacinia; 3) fémur con 2-3 setas apicales espatuladas; 4) uñas tarsales 0.5 veces la longitud del tarso (Domínguez et al., 2006). 
Material Examinado: CIUQ 8452A, P37.

Comentarios. Cloeodes posee 31 especies descritas para América del sur (Domínguez y Dos Santos, 2014; Salles et al., 2015). En Colombia se encuentra reportada la especie $C$. redactus para el Amazonas (Días et al., 2009). La identificación a nivel de especie no se pudo realizar debido al deterioro de los individuos

\section{Mayobaetis ellenae Mayo 1973}

Diagnosis: 1) Antena corta, 1.5 veces la longitud de la cabeza; 2) Escapo y pedicelo aplanados, escapo tres veces la longitud del pedicelo: 3) Cercos con una hilera de espinas en el margen externo; 4) Diseño de coloración del abdomen con una banda oscura a lo largo de los tergos IIVII o VIII.

Material Examinado: CIUQ 45D, P35; MAUQ 80A, P32; CIUQ 8397, P42

Comentarios. Esta especie fue descrita a partir de ninfas y adultos, se encuentra registrada para los países de Costa Rica, Ecuador y Perú (Domínguez et al., 2006); en Colombia se encuentra ampliamente distribuida, reportándose para Antioquia, Boyacá, Caldas, Cauca, Cundinamarca, Magdalena, Meta, Nariño, Putumayo, Quindío, Risaralda y Valle del Cauca (Días et al., 2009; Salinas et al, 2012; Salinas et al, 2017).

\section{Nanomis rasmusseni Chacón, Pescador \& Seg- nini 2013}

Diagnosis: 1) Margen anterior del labro con setas triangulares aplanadas (como con uñas); 2) Conjunto interno de incisivos de la mandíbula derecha con cinco dentículos; 3) Prosteca derecha bífida desde el tercio basa; 4) Margen posterior de los tergos abdominales I-VIII lisos, IX-X con espinas; 5) Branquias ligeramente asimétricas con la parte media sombreada de marrón; 6) Tráquea de las branquias no pigmentadas y con pocas ramificaciones laterales (Chacón et al., 2013).

Material examinado: MIUQ 469, P19
Comentarios. Esta especie fue originalmente descrita para Venezuela (Chacón et al., 2013). Recientemente fue registrada para Colombia (Salinas et al, 2018; Forero-Céspedes y ReinosoFlórez, 2018).

\section{Prebaetodes meridinensis Chacón, Pescador \& Segnini, 2010}

Diagnosis: 1) mandíbula derecha con ocho dentículos en los incisivos; 2) mandíbula izquierda con diez dentículos en los incisivos; 3) segmento II del palpo labial recto; 4) tibia con forma recta; 5) margen dorsal de la tibia con 5-8 setas como espinas (Chacón et al., 2010).

Material Examinado: MIUQ 684, P22; MAUQ, P35

Comentarios. Esta especie fue originalmente descrita para Venezuela (Chacón et al., 2010). Recientemente fue registrada para Colombia en la región andina (Salinas et al., 2019).

\section{Varipes Lugo-Ortiz \& Mccafferty, 1998}

Diagnosis: 1) Fémures I sin hilera transversa de setas como espinas; 2) Prosteca larga y bífida; 3) Uñas tarsales con dos hileras de dentículos 6 dentículos; 4) Margen posterior de los tergos abdominales con espinas (Domínguez et al., 2006). Material Examinado: MIUQ 387B, P25; 430B, P28.

Comentarios: Varipes posee seis especies descritas para América del sur (Domínguez y Dos Santos, 2014). En Colombia se encuentra reportada la especie $V$. lasiobrachius (Días et al. 2009). La identificación a nivel de especie no se pudo realizar debido al mal estado de los individuos.

\section{Zelusia principalis Lugo-Ortiz \& Mccafferty, 1998}

Diagnosis: 1) Labro con una hilera de setas robustas subapicales en cualquier lado de la línea media; 2) Maxila con el palpo dos veces la longi- 
tud de la galea-lacinia y segmento II 1.5 veces la longitud del segmento I; 3) Segmento II del palpo labial sin proyección lateral; 4) Uñas tarsales con una hilera de dentículos pequeños; 5) Pterotecas posteriores ausentes; 6) Margen posterior de los tergos abdominales con espinas irregulares; 7) Branquias presentes en los segmentos II-VII; 8) Filamento terminal subigual a la longitud del cerco (Domínguez et al., 2006).

Material Examinado: MIUQ 387B, P25; 430B, P28.

Comentarios. Esta especie es conocida a partir de ninfas. Actualmente se encuentra registrada para Colombia (Días et al., 2009).

\section{AGRADECIMIENTOS.}

Este trabajo recibió financiación de la Universidad del Quindío, vicerrectoría de investigaciones (proyecto 824) a C.R-V, Facultad de Ciencias Básicas y Tecnologías-programa de Biología y dentro del programa jóvenes investigadores de Universidad del Quindío y Colciencias. Cristian Román-P. (UA, Tucson, USA) reviso ambos resúmenes.

\section{REFERENCIAS}

1. Chacón, M.M., Pescador, M.L. \& Segnini S. 2010. The adult and redefinition of the genus Prebaetodes Lugo-Ortiz \& McCafferty (Ephemeroptera: Baetidae), with description of a new species from Venezuela. Aquatic Insects, 32: 143 157.https://doi.org/10.1080/01650424.2010.482940.

2. Chacón, M.M., Pescador, M.L \& Segnini S. 2013. New species of Nanomis. Lugo-Ortiz and McCafferty, 1999 (Ephemeroptera: Baetidae) from Venezuela. Aquatic Insects. International Journal of Freshwater Entomology, 35: 1-2, 1-14.

3. Chará, J., Zúñiga, M. C., Giraldo, L.P., Pedraza, G., Astudillo, M., Ramirez, L. \& Posso C.E. 2009. Diversidad y abundancia de macroinvertebrados acuáticos en quebradas de la cuenca del río La Vieja, Colombia. (pp. 129-142). En: Rodríguez, J.M., Camargo, J.C., Niño, J., Pineda, A.M., Arias, L.M., Echeverry, M.A. \& Miranda, C.L. (Eds.) Valoración de la Biodiversidad en la Ecorregión del Eje Cafetero. Centro de Investigación y Estudios en Biodiversidad y Recursos Genéticos, CIEBREG. Pereira, Colombia.

4. Dias, L.G.; Zuñiga, M.C. \& Bacca T. 2009. Estado Actual del Conocimiento del Orden Ephemeroptera en Colombia. In: Congreso Sociedad Colombiana de Entomología, $36^{\circ}$. Memorias. Colombia, Sociedad Colombiana de Entomología, p. 236-253.

5. Domínguez, E., Molineri, C.; Pescador, M.L., Hubbard, M.D. \& Nieto C. 2006. Diversidad Acuática en América Latina. Vol. 2: Ephemeroptera de América del Sur. Sofia-Moscú, Pensoft.

6. Domínguez, E., Molineri, C. \& Nieto C. 2009. Ephemeroptera. En E. Domínguez y H. R. Fernández (Eds.), Macroinvertebrados bentonicos sudamericanos. Sistemática y biología (pp. 55-93). Tucumán: Fundación Miguel Lillo.

7. Domínguez, E. \& Dos Santos D.A. 2014. Co-authorship network (and other contextual factors) behind the growth of taxonomy of South American Ephemeroptera: A scientometric approach. Zootaxa, 3754: 59-88.

8. Forero-Céspedes, A. M. \& Reinoso-Flórez, G. 2018. Primer registro de Nanomis rasmusseni Chacón, Pescador \& Segnini, 2013 (Insecta: Ephemeroptera: Baetidae) en el departamento del Tolima, Colombia. Revista de la Academia Colombiana de Ciencias Exactas Físicas y Naturales, 42: 230-231, julioseptiembre de 2018. doi: http://dx.doi.org/10.18257/raccefyn.590.

9. García-Alzate, C., P. A. Villegas-Acosta \& Román-Valencia C. 2017. Análisis Fisicoquímico y Biológico del Rio Santo Domingo, afluente Rio Verde, cuenca del Rio La Vieja, Alto Cauca, Colombia. Boletín Científico del Museo de Historia Natural, Univ. de Caldas, Manizales, Colombia, 21: 31-51.

10. Gattolliat, J.L. \& Nieto C. 2009. 'The family Baetidae (Insecta: Ephemeroptera): synthesisand future challenges. Aquatic Insects, 31: 41- 62.

11. Gutiérrez, Y \& Llano C. 2015. First record of Americabaetis alphus (Insecta: Ephemeroptera: Baetidae) from Colombia. Revista Colombiana de Entomología, 41: 147-148

12. Gutiérrez, C \& Reinoso G. 2010. Géneros de ninfas del orden Ephemeroptera (Insecta) del departamento del Tolima, Colombia: listado preliminar. Biota Colombiana, 11: 23-32.

13. Lugo-Ortiz, C.R. \& McCafferty W.P. 1999. Global Biodiversity of the Mayfly Family Baetidae (Ephe- 
meroptera): A generic perspective. Trends in Entomology, 2: 45-54.

14. Motta-Díaz, A, Nieto C \& Aranguren-Riaño N.J. 2012. New reports of the genus Baetodes Needham and Murphy (Ephemeroptera: Baetidae) from Colombia. Entomotropica, 27: 45-47.

15. Rozo, M.P. \& Salinas L.G. 2016. Inventario del orden Ephemeroptera (Insecta) en la quebrada Coquital, Serranía de Tripogadí, Departamento del Chocó, Colombia. Entomotropica, 31: 1-13.

16. Román-P, C. \& Román-Valencia C. 2017. Dieta y reproducción de Bryconamericus caucanus (Characiformes, Characidae) en la quebrada La Venada, rio Quindio, Alto Cauca, Colombia. Rev. MVZ Córdoba, 22: 6296-6309.

17. Ruiz-C., R.I., C. Román-Valencia \& C.H. Gonzales-E. 2018. Revisión de la gestión ambiental sobre el territorio y cambio climático en el departamento del Quindio, Colombia. Gestión y Ambiente, 22:137150.

18. Salinas, L.G., Días, L.G., Salles, F.F. \& Bacca T. 2011. Three new species of Baetodes Needham y Murphy (Ephemeroptera: Baetidae) from Colombia. Zootaxa, 3110: 61-68.

19. Salinas, L.G., Dias, L.G., Bacca, T., Zuñiga, M.C. \& Rodríguez M. 2012. Primeros registros de Ephemeroptera (Insecta) para el departamento de Putumayo, Colombia. Boletín Científico- Museo de Historia Natural, Univ. de Caldas, Manizales, Colombia, 16: 198-208.

20. Salinas, L.G., Rojas-Peñadiana, J.I., Osorio-Ramírez P. \& Caro-Caro C.I. 2017. New records of Ephemeroptera from the Colombian Orinoco river basin of the Meta department. Revista Colombiana de Entomología, 43: 271-276.

21. Salinas, L.G., Villegas, P.A \& Román-Valencia C. 2018. First record of the nymph of Nanomis rasmusseni Chacon, Pescador \& Segnini, 2013 (Ephemeroptera: Baetidae) from Colombia. Papéis Avulsos de Zoología, 58: e20185822. http://doi.org/10.11606/1807-0205/2018.58.22.

22. Salinas, L.G., Villegas, P.A., Salles, F.F \& Román-Valencia C. 2019. First record of Prebaetodes meridinensis Chacon, Pescador \& Segnini 2010 (Ephemeroptera: Baetidae) from Colombia. Check List, 15: $323-325$

23. Salles, F.F., Da-Silva E.R., Serrão J. E. \& Francischetti C.N. 2004. Baetidae (Ephemeroptera) na região sudeste do Brasil: novos registros e chave para os gêneros no estágio ninfal. Neotropical Entomology, 33: 725-735.

24. Salles, F.F., Massariol, F.C., Angeli, K.B., Lima, M.M., Gattolliat, J-L. \& Sartori M. 2015. Revealing the diversity of Cloeodes Traver, 1938 (Ephemeroptera: Baetidae) in the Neotropics: description of eleven new species from Brazilian mountain ranges. Zootaxa, 4020: 001-050.

25. Vilela Cruz, P., Boldrini, R., Quinto, C.F. \& Frontado H. 2012. New Baetidae (Insecta: Ephemeroptera) Records from Venezuela and nymph description of an unnamed Fallceon species. International Journal of Zoology, 2012, Article ID 837092, 6 pages. doi:10.1155/2012/837092

26. Vilela Cruz, P., Salles, F.F. \& Hamada N. 2014. Callibaetis Eaton (Ephemeroptera: Baetidae) from Brazil, Journal of Natural History, DOI: 10.1080/00222933.2013.791883.

27. Vilela Cruz, P., Salles, F.F. \& Hamada N. 2017. Four new species of Callibaetis Eaton (Ephemeroptera: Baetidae), nymphal description of Callibaetis (Abaetetuba) fasciatus (Pictet) and keys for South American species of Callibaetis. Zootaxa, 4250: 229-261.

28. Vinasco-Mondragón, A.F. \& Zúñiga M.C. 2016. Primeros registros de Callibaetis radiatus y C. viviparus (Ephemeroptera: Baetidae) para Colombia. Revista Colombiana de Entomología, 42: 91-94.

29. Waltz, R.D. \& Mccafferty W. P. 1987. Revision of the genus Cloeodes Traver (Ephemeroptera: Baetidae). Annales. Entomological Society American, 80: 191-207.

30. Zúñiga, M.C., Chará, J., Giraldo, L.P., Chará-Sernay A.M. \& Pedraza G.X. 2013. Composición de la comunidad de macroinvertebrados acuáticos en pequeñas quebradas de la región andina colombiana, con énfasis en la entomofauna. Dugesiana, 20: 263-277. 